\title{
FROM THE EDITOR Spirituality — The psyche or the soul?
}

The Divine Spirit hovered upon the surface of the water

Genesis, 1:2

The medical literature is conflicted and uncertain when discussing spirituality. "Spiritual" was once clearly understood when religion was widely accepted in the West and the existence of the soul was assumed. (Harper, 2011; Egan, 2011) The medical literature today does not refer to the classical concept of spirituality. One has a sense that modern medicine is embarrassed by concepts such as the soul and immortality. However, in recent years the palliative care, psycho-oncology, and (even) oncology literatures have re-explored the spiritual concept, albeit in a secular guise. The results are in my opinion disappointing.

Salander (2006) vigorously critiqued the academic use of the word spiritual and pointed out serial misuses in the literature. He objected to spiritual being conflated with the concepts religion, meaning, and existential (and one might add emotion, psychology, and hope). Furthermore, Salander challenges (correctly in my view) papers that mislabel psychosocial issues as spiritual ones. He noted that the concept of "spirituality" as currently portrayed in the literature has no theoretical basis (presumably in the absence of the concept of the soul) and can mean many things to many people.

It is probable that the medical literature is changing the use and meaning of the word "spiritual" in response to changing culture. However, is it a mistake to reject the phenomenon that the original meaning of the word described, namely the spiritual experience associated with religiousness, mysticism, and the soul? Has the original spiritual experience associated with the soul vacated the human condition?

\section{DEFINITIONS OF SPIRITUALITY}

Commentators pay lip service to distinguishing between spirituality and emotions. The Functional Assessment of Chronic Illness Therapy - Spiritual
Well-Being - 12-item Scale (FACIT-sp-12), used in many articles, proffers little insight in exploring spirituality (Cella, 2010). It is based on circular questions, and merely confirms that "meaning, purpose, peace, and calm" are important to people. The items include the following (Cella, 2010): "Below is a list of 12 statements that other people with your illness have said are important. Please circle or mark one number (from 0-4) per statement to indicate your response as it applies to the past seven days: "I feel peaceful; I have a reason for living; My life has been productive; I have trouble feeling peace of mind; I feel a sense of purpose in my life; I am able to reach down deep into myself for comfort; I feel a sense of harmony within myself; My life lacks meaning and purpose; I find comfort in my faith or spiritual beliefs; I find strength in my faith or spiritual beliefs; My illness has strengthened my faith or spiritual beliefs; I know that whatever happens with my illness, things will be okay."

What does an objective measure of "spiritual" measure when it asks in $25 \%$ of the questions: are you spiritual? The fact that it has been validated does not convince that its intellectual provenance is valid. There is really no room in academic discussion to avoid a definition. Sometimes there is a set of characteristics that have not coalesced sufficiently, in order to achieve the high degree of integrity required to be labeled a definition. Sometimes we have to be pragmatic and make do with a "good enough" set of descriptors as supported by the evidence. The FACIT-sp-12 does not replace a definition, nor is it "good enough."

The Canadian Hospice Palliative Care Association defines spirituality as: "An existential construct inclusive of all the ways in which a person makes meaning and organizes his/her sense of self around a personal set of beliefs, values and relationships. This is sometimes understood in terms of transcendence or inspiration. Involvement in a community of faith and practice may or may not be a part of an individual's spirituality" (Canada Hospice Association, 2004). Another palliative care definition states: 
"Spirituality ... moves the individual towards knowledge, love, meaning, peace, hope, transcendence, connectedness, compassion, wellness and wholeness ... It is an innate tendency toward meaning it is expressed in an individual's search for ultimate meaning through participation in religion and/or belief in God, family, naturalism, rationalism, humanism, and the arts" (Bryson, 2004). Clinical chaplains suggest: "Spirituality is my being: my inner person. It is who I am - unique and alive. It is my body, my thinking, my feelings, my judgments, and my creativity. My spirituality motivates me to choose meaningful relationships and pursuits (Harper, 2011).” A United States national conference used a consensus process to formalize the definition of spirituality: "(Spirituality) is the aspect of humanity that refers to the way individuals seek and express meaning and purpose and the way they experience their connectedness to the moment, to self, to others, to nature, and to the significant or sacred" (Puchalski, 2009).

Rodin's group in Toronto (Lo, 2011) concluded a longitudinal study of spirituality in 747 advanced cancer patients over eight years, by stating "we have demonstrated that spiritual well-being is supported not only by religious belief systems, but also by self-worth and the sense of connection with others, and is disrupted by physical suffering." And: that "spiritual well-being has come to be regarded as a psychological outcome indicating the extent to which individuals are at peace with themselves, feel their lives have meaning and purpose, and derive comfort from their beliefs in the face of suffering." From New Zealand a comprehensive article gave an honest if ultimately confusing definition: "Spirituality means different things to different people. It may include (a search for): one's ultimate beliefs and values; a sense of meaning and purpose in life; a sense of connectedness; identity and awareness; and for some people, religion. It may be understood at an individual or population level" (Egan, 2001).

Spirituality according to these authors incorporates many aspects of human psychology. These definitions ultimately fail because they are imprecise and over-inclusive. Many of the words overlap one another conceptually and are unhelpful in identifying what is unique and characteristic about spiritual. Undefined and vaguely used words ("my being," "wellness and wholeness," "naturalism," "meaning," "in the face of suffering," "at peace," "authenticity, dignity, and hope") are un-tested (if not un-testable). Furthermore, they are at risk of discarding the original and still extant phenomenon that spiritual formerly described. The spectrum and imprecision of definitions confirms the absence of a rigorous theoretical base and undoubtedly contributed to Salander's intellectual dyspepsia.

\section{AN HISTORICAL PERSPECTIVE ON SPIRITUALITY}

Spirituality today is orphaned between the brain and God - I shall explain. But before that, I suggest that the main problem today, is not so much separating spiritual from religious, but rather distinguishing spiritual from emotional since there are overlapping features.

\section{Brain}

Much work has been done on the nature of consciousness. It is still poorly understood in neuro-chemical and neuro-anatomical terms. For example, it is not understood how anesthetic agents render unconsciousness (Mashour, 2005). Nor is there an understanding how, if one particular area of the brain "lights up," suddenly the whole brain can experience a unified awareness (Greenfield, 1998). However, there is a consensus among researchers about two things, albeit related. First, that the mind is the brain. That is, the mind, i.e., consciousness, is due solely to the brain's neurophysiology, and when the brain ceases, the mind ceases (Denton, 2005). The second point of agreement in the consciousness literature is that there is no independent soul or spirit that animates the brain. Since there is no spiritual entity separate from the brain, all thoughts and psychological activities that we are aware of, are due only to the activity of the brain's neuro-transmitters. Thus, in the absence of the soul, the challenge is to distinguish what we mean by spiritual and what by emotional, keeping in mind (as it were) that both are products of the brain and its neuro-transmitters.

\section{God}

People who believe in a biblical version of the creation of man subscribe to the model that there is a separate entity which resides in the body called the soul (created by God) which animates the person and is the source of spirituality, including character and personality. The soul, being indestructible, outlives the death of the brain and returns to its Maker. This model tends to equate consciousness (the mind) with the soul. The existence of a soul makes for a simpler model of spirituality. Nevertheless one is still left with the problem of distinguishing phenomenologically between spirituality and emotions. Today most people (including spirituality researchers) speak of a "secular" spirituality and avoid the need to use the "soul" hypothesis.

\section{A NEW DEFINITION OF SPIRITUALITY}

I propose a concise definition of secular spirituality which to be sure includes elements mentioned by 
other authors. However, it distills out the two essential characteristics. It is not predicated on the existence of a soul, it assumes that the brain is the mind, and that the spiritual experience is a manifestation of the brain's neuro-transmitters.

The spiritual experience is characterized by two features: (1) there is an experience of unification (or unity); (2) this sense of unification (or unity) is accompanied by a change in the state of consciousness. I shall discuss each point in turn.

\section{Unification}

Nearly 100 years ago Freud, quoting a friend, suggested that a spiritual experience can be independent of religious dogma: "It is a feeling which he would like to call a sensation of "eternity," feeling as of something limitless, unbounded, something "oceanic" ... a purely subjective experience, not an article of belief ... it is the source of the religious spirit ... One may rightly call oneself religious on the grounds of this oceanic feeling alone, even though one rejects all beliefs and all illusions ... So it is a feeling of indissoluble connection, of belonging inseparably to the external world as a whole" (Freud, 1930).

Today we could describe this as a spiritual experience of the secular variety. Note that the two key experiences are inextricably linked. The "oceanic feeling" and the "indissoluble connection" are combined to form the basis of this "religious" experience. Freud noted of himself: "I myself cannot discover this 'oceanic' feeling in myself," although he goes on to acknowledge that "the state of being in love threatens to obliterate the boundaries between the ego and the object." (Gratefully Freud refers to being in love as "an unusual state, it is true, but not one that can be judged as pathological.") And this was the closest Freud came, he admitted, to understanding an "oceanic feeling." Several of the definitions quoted, use the word "connectedness" which approximates to "unification" and "the indissoluble connection." Salander argued emphatically that a spiritual experience is irrevocably linked to religion and/or culture (Salander, 2006). I do not think it is necessarily so. I think we can make the argument for a secular spirituality, as did Freud.

\section{Change in State of Consciousness}

What is meant by an altered state of consciousness? While consciousness does not have an agreed upon definition, it has defining characteristics. For example, consciousness is unity. A given conscious state cannot be divided into smaller components. Furthermore, consciousness is mutually exclusive, meaning we cannot be aware of two scenes or thoughts or activities at the same time. This is readily demonstrated by examining Rubin's vase, the ambiguous black and white picture which is either a vase or the profiles of two people. One cannot be aware of both images at the same time. One flicks rapidly between the vase and the faces - either, or; unity or unity (Rubin, 1915).

The intensity of the conscious experience varies within each individual from day to day, even moment to moment (since the minimum length of a given conscious state is about 150 milliseconds) (Greenfield, 1998). Dreaming, or consciously observing the passage of time, or being engrossed in a book, are different states of consciousness. People experience altered states of consciousness with and without drugs. "Altered" in our sense of a spiritual experience means a more intense and focused, and less distractible awareness. Meditation, concerts, sporting events, love, or a profound cognitive insight - all can achieve a conscious experience that is qualitatively different from a regular state of awareness and might in certain circumstances be called a spiritual experience or transcendence. However, since the brain is the mind, it still falls under the rubric of a neuro-chemical event. Freud's "oceanic feeling" is I suggest a changed state of consciousness which is characterized by a sense of unity (or "indissoluble connection").

\section{CONCLUSION}

Most people with cancer relate to their ill-mood (depression, anxiety, distress, anger) as emotional or existential problems - not spiritual ones. In fact, for most oncologists and palliative medicine physicians, spiritual is not part of their lexicon, at least at the bed-side. How do we clinically separate an emotional experience from a spiritual one? Emotions, such as joy, sadness, anger, and fear tend to be self-contained, directly correlated, and in proportion to the cause. An emotion tends to be a self-referential inner-directed experience, without an "oceanic feeling" of connecting to or fusing with other. Thus, I experience emotional joy at my child's birthday. Generally this would not be called spiritual.

Spiritual experiences seem always to be described as positive, whereas emotions can be positive and negative. Spiritual experiences tend to be exponentially greater than the initiating experience. Emotions however can encourage a connection/ unification with Other. It might only be a matter of degree, or it might arise in a different part of the brain, with a different set of neuro-transmitters. Can an emotion become so intense that it develops spiritual characteristics? Probably. Alternatively, is there a spectrum between an emotion and a spiritual 
experience? Possibly. What happens with the neurotransmitters? No one knows.

After experiencing a massive left cortical hemorrhage, Jill Bolte Taylor, a neuro-scientist, observed: "My consciousness no longer retained the discriminatory function of my dominant analytical left brain ... Without the traditional sense of my physical boundaries, I felt that I was at one with the vastness of the universe ... my consciousness dwelled in a flow of sweet tranquility ... All I could perceive was right here, right now, and it was beautiful ... Now released from that restrictive circuitry, my right hemisphere relished in its attachment to the eternal flow. I was no longer isolated and alone. My soul was as big as the universe and frolicked with glee in a boundless sea ... Based upon the experience of losing my left mind, I wholeheartedly feel that the feeling of deep inner peace is neurological circuitry located in our right brain" (Taylor, 2006). Herein is a suggested neuro-chemical basis for distinguishing between an emotion and a spiritual experience. The neurotransmitters (different ones or combinations thereof) in different parts of the brain sub-serve the different subjective experiences.

The secular spiritual experience is a psychological phenomenon, not predicated on the existence of a soul, yet still retains the characteristic spiritual experience of a religious experience. Its two defining features are: (1) there is an awareness of unification or becoming one, with something other than self, and (2) it involves a specific and characteristic changed state of consciousness, distinct from an emotion. It is my view that this is a better hypothesis with which to research spirituality.

\section{REFERENCES}

Bryson, K.A. (2004). Spirituality, meaning and transcendence. Palliative and Supportive Care, 2, 321-328

Canadian Hospice Palliative Care Association. (2009). A Model to Guide Hospice Palliative Care. Appendices: Lexicon of Commonly Used Terms. http://www.chpca.net/ resource_doc_library/model_to_guide_hpc/Appendicesetc.pdf (Accessed June 21, 2009).

Cella, David. (2010). FACIT-Sp-12: Functional assessment of chronic illness therapy - Spiritual well-being; The 12-item spiritual well-being scale http://www.facit.org/ FACITOrg/Questionnaires (Accessed March 13, 2014).

Denton, D. (2005). The Primordial Emotions: The Dawning Of Consciousness. Oxford: Oxford University Press.

Egan, R., MacLeod, R., Jaye, C., et al. (2011). What is spirituality? Evidence from a New Zealand Hospice Study. Mortality, 16, 307-324

Freud, Sigmund. (1957). Civilization and Its Discontents, London: The Hogarth Press Limited, pp. 1-22.

Harper, J.M. \& Rudnick, J.E. (2011). The Role of the Chaplain in palliative care. In Oxford Textbook of Palliative Medicine. pp. 197-205.

Lo, C., Zimmermann, C., Gagliese, L., et al. (2011). Sources of spiritual well-being in advanced cancer. British Medical Journal Supportive and Palliative Care, 1, 149-153

Mashour, G.A. (2006). Integrating the science of consciousness and anesthesia. Anesth Analg, 103, 975-982.

Puchalski, C., Ferrell, B., Virani, R., et al. (2009). Improving the quality of spiritual care as a dimension of palliative care: The report of the consensus conference. Journal of Palliative Medicine, 12, 885-904.

Rubin, Edgar. (1915). Rubin's Vase. http://en.wikipedia. org/wiki/Rubin_vase (Accessed March 13, 2014).

Salander, P. (2006). Who needs the concept of "spirituality"? Psycho-Oncology, 15, 647-649.

Taylor, Jill Bolte. (2006). My Stroke of Insight. New York: Plume Publication, pp. 63-71, 168.

SIMON WEIN, MD Co-Editor 